\title{
Cerebrospinal Fluid Levels of $\beta$-Amyloid 40 and $\beta$-Amyloid 42 are Proportionately Decreased in Amyloid Positron-Emission Tomography Negative Idiopathic Normal-Pressure Hydrocephalus Patients
}

\author{
Hyun Jae Kim ${ }^{\mathrm{a}}$ \\ Tae Sung Lima \\ Sun Min Lee ${ }^{a}$ \\ Tae-Sung Kim ${ }^{\mathrm{b}}$ \\ Youngbin $\mathrm{Kim}^{\mathrm{C}}$ \\ Young-Sil $A n^{d}$ \\ Young Chul Youn ${ }^{\mathrm{e}}$ \\ Sun Ah Park ${ }^{\mathrm{a}, \mathrm{f}}$ \\ Jaerak Chang ${ }^{\text {b,c }}$ \\ So Young Moon ${ }^{\mathrm{a}}$ \\ aDepartments of Neurology, \\ ${ }^{b}$ Brain Science, ${ }^{\mathrm{C} B i o m e d i c a l ~ S c i e n c e s, ~}$ \\ 'Nuclear Medicine and Molecular Imaging, \\ and ${ }^{\mathrm{f}}$ Anatomy, Ajou University \\ School of Medicine, Suwon, Korea \\ eDepartment of Neurology, \\ Chung-Ang University \\ College of Medicine, Seoul, Korea
}

\begin{abstract}
Background and Purpose Cerebrospinal fluid (CSF) biomarkers of Alzheimer's disease (AD) could be misleading in idiopathic normal-pressure hydrocephalus (iNPH). We therefore investigated the CSF biomarkers in 18F-florbetaben amyloid-negative positron-emission tomography (PET) [amyloid PET(-)] iNPH, amyloid-positive PET [amyloid PET(+)] AD, and cognitively normal $(\mathrm{CN})$ subjects.

Methods Ten amyloid PET(+) AD patients (56.7 \pm 5.6 years old, mean \pm standard deviation), 10 amyloid PET(-) iNPH patients (72.8 \pm 4.5 years old), and $8 \mathrm{CN}$ subjects (61.2 \pm 6.5 years old) were included. We measured the levels of $\beta$-amyloid $(\mathrm{A} \beta) 40, \mathrm{~A} \beta 42$, total tau (t-tau) protein, and phosphorylated tau (p-tau) protein in the CSF using enzyme-linked immunosorbent assays.

Results The level of $A \beta 42$ and the $A \beta 42 / A \beta 40$ ratio in the CSF were significantly lower in $\mathrm{AD}$ than in iNPH or $\mathrm{CN}$ subjects. The $\mathrm{A} \beta 40$ level did not differ significantly between $\mathrm{AD}$ and iNPH ( $p=1.000)$, but it did between AD and CN subjects ( $p=0.032$ ). The levels of both t-tau and p-tau were higher in $\mathrm{AD}$ than in $\mathrm{iNPH}$ or $\mathrm{CN}$ subjects. The levels of $\mathrm{A} \beta 42, \mathrm{~A} \beta 40, \mathrm{t}$-tau, and p-tau were lower in iNPH than in $\mathrm{CN}$ subjects, but there was no significant difference after controlling for age.

Conclusions Our results suggest that the mechanism underlying low CSF A $\beta$ levels differs between amyloid PET(-) iNPH and amyloid PET(+) AD subjects. The lower levels of all CSF biomarkers in iNPH patients might be due to reduced clearances from extracellular fluid and decreased brain metabolism of the periventricular zone in iNPH resulting from glymphatic dysfunction.
\end{abstract}

Key Words Alzheimer's disease biomarkers, idiopathic normal pressure hydrocephalus, amyloid positron-emission tomography, cerebrospinal fluid.
Received December 18, 2018

Revised February 14, 2019

Accepted February 14, 2019

\section{Correspondence}

So Young Moon, MD, $\mathrm{PhD}$

Department of Neurology,

Ajou University School of Medicine,

164 World cup-ro, Yeongtong-gu,

Suwon 16499, Korea

Tel +82-31-219-5175

Fax +82-31-219-5178

E-mailssymoon.bv@gmail.com

Jaerak Chang, $\mathrm{PhD}$

Department of Brain Science,

Ajou University School of Medicine,

164 World cup-ro, Yeongtong-gu,

Suwon 16499, Korea

Tel +82-31-219-4477

Fax +82-31-219-5178

E-mail jaerakchang@ajou.ac.kr

\section{INTRODUCTION}

Idiopathic normal-pressure hydrocephalus (iNPH) is characterized by the clinical triad of symptoms of cognitive impairment, gait difficulty, and urinary incontinence, and is accompanied by ventricular enlargement in brain imaging. ${ }^{.}$Shunt surgery is thought to alleviate these symptoms in some iNPH patients, but other coexisting neurodegenerative diseases compromise the effectiveness of this approach and thus should be investigated thoroughly before surgery. Coexisting Alzheimer's disease (AD) has been reported in $30-60 \%$ of iNPH patients and is associated with poor shunt response and progressive cognitive decline after shunt surgery. ${ }^{2,3} \mathrm{AD}$ is usually evaluated by analyzing the levels of $\beta$-amyloid $(\mathrm{A} \beta) 42$, total

(®) This is an Open Access article distributed under the terms of the Creative Commons Attribution Non-Commercial License (https://creativecommons.org/licenses/by-nc/4.0) which permits unrestricted non-commercial use, distribution, and reproduction in any medium, provided the original work is properly cited. 
tau (t-tau) protein, and 181-threonine-phosphorylated tau (p-tau) protein in the cerebrospinal fluid (CSF) using enzymelinked immunosorbent assays (ELISAs). ${ }^{4}$ Low A $\beta 42$ levels are thought to reflect brain amyloid deposition, while high t-tau and $\mathrm{p}$-tau levels indicate neurodegeneration. Among these CSF biomarkers, the change in $\mathrm{A} \beta 42$ levels occurs earlier than those in the other two tau-associated markers. A specific decrease in $\mathrm{A} \beta 42$ combined with no change in $\mathrm{t}$-tau and $\mathrm{p}$-tau levels has been considered a preclinical marker for coexisting $\mathrm{AD}$ in $\mathrm{iNPH}$ patients, without involving any substantial neuronal injury. ${ }^{5-8}$

Some researchers have recently reported that CSF AD biomarkers may be misleading in iNPH patients. ${ }^{9}$ A recent meta-analysis suggested that iNPH is associated with significantly low CSF levels of $\mathrm{A} \beta 42$, $\mathrm{t}$-tau, and $\mathrm{p}$-tau. ${ }^{10}$ Various hypotheses have been proposed for explaining the reduced levels of $\mathrm{AD}$ biomarkers in iNPH. One hypothesis is that tight sulci over the cortex of iNPH patients could compromise the convective flow of interstitial fluid and the clearance of amyloid precursor protein (APP) fragments. ${ }^{9}$ Other authors have proposed that reduced periventricular metabolism accompanied by decreased levels of APP-derived proteins but no major cortical degeneration could explain these results. ${ }^{6}$ However, these studies did not include $18 \mathrm{~F}$-florbetaben amyloid positronemission tomography (PET) analyses, which probably affected the interpretation of the levels of CSF AD biomarkers in iNPH patients.

The aim of the present study was to measure CSF AD biomarkers in amyloid-positive PET [amyloid PED(+)] AD, amyloid-negative PET [amyloid PED(-)] iNPH, and cognitively normal $(\mathrm{CN})$ subjects, and identify the mechanisms underlying the observed changes.

\section{METHODS}

\section{Subjects}

Ten probable-iNPH and 10 probable-AD patients were prospectively recruited at Ajou University Hospital (Suwon, Korea) from March 2015 to February 2017. Eight CN subjects were recruited at Chung-Ang University Hospital (Seoul, Korea). All three groups were recruited using convenience sampling. The selection criteria for probable-iNPH patients included the following: 1 ) insidious onset at an age $\geq 40$ years, disease duration $\geq 6$ months, progressive disease course, and no other neurological, psychiatric, or medical conditions other than the expected disease symptoms; 2) ventricular enlargement that was not entirely attributable to cerebral cortical atrophy, as detected by magnetic resonance imaging (MRI); 3) gait disturbance with or without cognitive deficits or urinary disturbances; and 4) no evidence of high intracranial pressure $\left(70-245 \mathrm{~mm} \mathrm{H}_{2} \mathrm{O}\right) .{ }^{11}$ The Evans' index was assessed using 3-Tesla MRI (Philips 3.0T Achieva; Best, the Netherlands), and defined as the maximal frontal horn ventricular width divided by the transverse inner diameter of the skull. This index is indicative of ventriculomegaly if it is $\geq 0.3{ }^{11}$

The clinical syndrome in patients with iNPH was characterized and graded using a NPH scale modified from Larsson et al. ${ }^{12}$ and Krauss et al. ${ }^{13}$ that assessed gait $[1=$ normal, $2=$ walk without any assistive device but insecure, $3=$ walk with cane, $4=$ walk with bimanual support (walker), $5=$ =walk with an assistant, and $6=$ wheelchair-bound], urinary disturbance $(0=$ normal, $1=$ sporadic incontinence or urge phenomena, $2=$ frequent incontinence or urge phenomena, and $3=$ no or minimal control of bladder function), and cognitive deficit ( $0=$ normal, $1=$ minimal attention or memory deficits, $2=$ consider able attention or memory deficits but oriented to situational context, and $3=$ not or only marginally oriented to situational context). Patients were evaluated before performing a lumbar puncture (LP).

All probable- $\mathrm{AD}$ patients were selected based on the criteria proposed by the National Institute of Neurological and Communicative Diseases and Stroke and the Alzheimer's Disease and Related Disorders Association. ${ }^{14}$ In patients with iNPH and $\mathrm{AD}$, white-matter hyperintensities (WMH) were assessed using the Clinical Research Center for Dementia of South Korea (CREDOS) rating scale. ${ }^{15}$ We excluded patients with a history of significant hearing or visual impairments that could interfere with interviews, a history of other neurological disorders (e.g., idiopathic Parkinson's disease, dementia with Lewy bodies, or active epilepsy), psychiatric illnesses (e.g., schizophrenia, mental retardation, major depression, or mania), or significant alcohol and/or other substance abuse, as well as patients taking psychotropic medications. $\mathrm{CN}$ subjects were chosen if they scored higher than the cutoff value in each cognitive domain test. The cutoff value for each test score was one standard deviation below the published norm for the age and education group. ${ }^{16}$

All patients provided written informed consent unless they had impaired decisional capacity, in which case caregivers provided consent and patients provided assent. This study was approved by the Ajou Institutional Review Board (AJIRBBMR-OBS-15-262).

\section{Sample collection}

All iNPH subjects underwent LP in the L3-4 or L4-5 spinal vertebrae interspace between 10 a.m. and 12 p.m. to drain $30-50 \mathrm{~mL}$ of CSF. During the procedure, $10-\mathrm{mL}$ aliquots of CSF were collected in polypropylene tubes after discarding the first 3-4 mL. Bloody or cloudy samples were not used. $\mathrm{AD}$ and $\mathrm{CN}$ subjects underwent $\mathrm{LP}$ using the same protocol 
except for CSF drainage. No serious adverse events were reported. The CSF samples were immediately centrifuged for $15 \mathrm{~min}$ at 2,000 $\mathrm{g}$ to remove cells, and the obtained supernatants were stored in polypropylene tubes and immediately frozen at $-80^{\circ} \mathrm{C}$ until being analyzed.

\section{ELISA}

The $A \beta 42$ and $A \beta 40$ levels in the CSF were measured using a sandwich ELISA method (INNOTEST A $\beta 42$ and $A \beta 40$, Fujirebio, Ghent, Belgium) specifically designed for measuring each $A \beta$ type, in accordance with the manufacturer's instructions. The CSF t-tau level was determined using a sandwich ELISA method (INNOTEST hTAU-Ag, Fujirebio) specifically designed to measure all tau isoforms irrespective of their phosphorylation status. The CSF p-tau level was measured at position 181 using a sandwich ELISA method [INNOTEST Phospho-Tau $\left({ }^{181} \mathrm{P}\right)$, Fujirebio] specifically designed to measure threonine p-tau. All biomarker levels were measured in duplicate and in accordance with the manufacturer's instructions.

\section{Amyloid PET}

All iNPH and AD subjects underwent ${ }^{18} \mathrm{~F}$-florbetaben PET. Brain PET/computed tomography (CT) images were obtained with a Discovery ST scanner (GE Healthcare, Milwaukee, WI, USA). An average of $300 \mathrm{MBq}$ of ${ }^{18} \mathrm{~F}$-florbetaben was injected intravenously, and scanning was initiated $90 \mathrm{~min}$ later. A noncontrast brain CT scan was performed in the automatic mode $(120 \mathrm{kV}, 30-100 \mathrm{~mA}$, and section width= $3.75 \mathrm{~mm}$ ) for attenuation correction, and was immediately followed by PET imaging in the three-dimensional mode for $20 \mathrm{~min}$. Motion artifacts were minimized by immobilizing the subject's head in a head holder. PET images were obtained by iterative reconstruction using an ordered subset expectation maximization algorithm with 4 iterations and 21 subsets.

\section{Interpretation and analysis of amyloid PET scans}

A board-certified nuclear medicine physician and neuroPET expert reviewed the amyloid PET images on an Advantage workstation (Advantage Sim, version 4.4, GE Healthcare). The examiners were blinded to all clinical data related to the patients. Grayscale transaxial brain images were visually assessed, and the regional uptakes of the tracer in the lateral temporal, frontal, and posterior cingulate cortex/precuneus, as well as in the parietal cortex were scored using previously described criteria $^{17}$ ( $1=$ no $A \beta$ load, $2=$ minor $A \beta$ load, and $3=$ significant $A \beta$ load). A score of 1 was interpreted as an amyloid PET(-) scan, and a score of 2 or 3 was interpreted as an amyloid PET(+) scan.
${ }^{18} \mathrm{~F}$-florbetaben uptake in the brain was quantified using recent T1-weighted MRI scans (MRI ${ }^{18} \mathrm{~F}$-florbetaben scan interval $=1.3 \pm 1.8$ years, mean \pm standard deviation). All MRI and PET images were processed using the PMOD Neuro tool (PNEURO, version 3.7, PMOD Technologies, Zürich, Switzerland). Automated segmentation was applied to the MRI images to remove the white matter and CSF. The segmented MRI scans and the respective PET images were coregistered and then spatially normalized according to the standard Montreal Neurological Institute T1 template. An automated anatomic labeling atlas ${ }^{18}$ was subsequently applied. The mean cortical standardized uptake value ratio (SUVR) was calculated in selected regions of interest including the frontal, lateral temporal, parietal, and posterior cingulate cortices, as assessed by visual scoring criteria. The PET images were analyzed quantitatively as described previously. ${ }^{19}$

\section{Statistical analyses}

The Kolmogorov-Smirnov test was used to determine whether continuous variables conformed to a normal distribution. Welch's analyses of variance with Games-Howell post-hoc tests were performed, since the assumption of variance homogeneity was not met in Levene's test. Fisher's exact test was used to compare dichotomous variables. The levels of CSF biomarkers were compared across the three groups using a univariate general linear model while adjusting for age. All statistical analyses were performed using SPSS (version 18.0, SPSS Inc., Chicago, IL, USA), and statistical significance was considered to be present at $p<0.05$.

\section{RESULTS}

The demographic and clinical characteristics of all of the study subjects are presented in Table 1 . The iNPH patients were significantly older $(72.8 \pm 4.5$ years) than the $\mathrm{AD}$ patients (56.7 \pm 5.6 years, $p<0.001)$ and $\mathrm{CN}$ subjects $(61.2 \pm 6.5$ years, $p=0.003$ ). The symptom scores in patients with iNPH were $2.0 \pm 0.0$ for gait, $1.6 \pm 0.5$ for urinary disturbance, and 1.6 \pm 0.5 for cognitive deficit. The degree of WMH was moderate in two iNPH patients and one AD patient, and mild in all of the other patients. Visual assessments of the amyloid PET scans revealed that the $10 \mathrm{AD}$ subjects were amyloid PET(+) and the $10 \mathrm{iNPH}$ subjects were amyloid PET(-). The cortical SUVR was significantly higher in AD patients $(1.97 \pm 0.25)$ than in iNPH patients $(1.37 \pm 0.16, p<0.001)$.

The levels of the assessed AD biomarkers in the CSF are described in Table 2 and plotted in Fig. 1. The CSF A $\beta 42$ level was significantly lower in $\mathrm{AD}$ patients than in $\mathrm{iNPH}$ patients and $\mathrm{CN}$ subjects $(p<0.001$ and $p<0.004$, respectively; Fig. 1A). The CSF A 440 level was also significantly lower in 
$\mathrm{A} \beta 40$ and $\mathrm{A} \beta 42$ in Amyloid Negative iNPH

Table 1. Demographic and clinical characteristics of the subjects

\begin{tabular}{lcccccc}
\hline & iNPH $(\boldsymbol{n}=\mathbf{1 0})$ & AD $(\boldsymbol{n}=\mathbf{1 0})$ & CN $(\boldsymbol{n}=\mathbf{8})$ & $\boldsymbol{p 1}$ & $\boldsymbol{p 2}$ & $\boldsymbol{p 3}$ \\
\hline Age (years) & $72.8 \pm 4.5$ & $56.7 \pm 5.6$ & $61.2 \pm 6.5$ & $<0.001$ & 0.003 & 0.294 \\
Sex (male/female) & $7 / 3$ & $4 / 6$ & $2 / 6$ & 0.370 & 0.153 & 0.638 \\
Education (years) & $10.6 \pm 3.4$ & $10.4 \pm 4.9$ & $13.0 \pm 2.4$ & 0.990 & 0.185 & 0.286 \\
K-MMSE score & $20.2 \pm 2.9$ & $12.4 \pm 4.2$ & $28 \pm 2$ & $<0.001$ & $<0.001$ & $<0.001$ \\
CDR & $0.85 \pm 0.47$ & $1.15 \pm 0.62$ & $0.37 \pm 0.23$ & 0.465 & 0.038 & 0.009 \\
CDR-SB & $4.15 \pm 3.38$ & $6.80 \pm 3.35$ & $0.94 \pm 0.32$ & 0.211 & 0.036 & 0.001 \\
Evans' index & $0.37 \pm 0.04$ & $0.26 \pm 0.03$ & - & $<0.001$ & - & - \\
Cortical SUVR & $1.37 \pm 0.16$ & $1.97 \pm 0.25$ & - & $<0.001$ & - & - \\
\hline
\end{tabular}

Data are mean \pm standard-deviation values.

AD: Alzheimer's disease, CDR: Clinical Dementia Rating, CDR-SB: CDR Sum of Boxes, CN: cognitively normal, iNPH: idiopathic normal-pressure hydrocephalus, K-MMSE: Korean version of the Mini Mental State Examination, p1: iNPH vs. AD, p2: iNPH vs. CN, p3: AD vs. CN, SUVR: standardized uptake value ratio.

Table 2. AD biomarker levels in the cerebrospinal fluid

\begin{tabular}{lcccrrr}
\hline & iNPH & AD & CN & p1 & p2 & p3 \\
\hline A $\beta 42(\mathrm{pg} / \mathrm{mL})$ & $892.8 \pm 311.4$ & $391.8 \pm 120.2$ & $1330.0 \pm 99.7$ & $<0.001$ & 0.089 & $<0.001$ \\
A $\beta 40(\mathrm{pg} / \mathrm{mL})$ & $5504.0 \pm 2867.1$ & $5462.5 \pm 2192.2$ & $9179.6 \pm 2596.9$ & 1.000 & 0.082 & 0.032 \\
A $\beta 42 / \mathrm{A} \beta 40$ ratio & $0.187 \pm 0.068$ & $0.077 \pm 0.020$ & $0.155 \pm 0.044$ & 0.001 & 0.178 & 0.003 \\
t-tau $(\mathrm{pg} / \mathrm{mL})$ & $238.9 \pm 103.7$ & $720.7 \pm 220.1$ & $294.5 \pm 95.1$ & $<0.001$ & 1.000 & $<0.001$ \\
p-tau $(\mathrm{pg} / \mathrm{mL})$ & $30.4 \pm 9.5$ & $78.1 \pm 30.6$ & $36.2 \pm 11.1$ & 0.001 & 0.560 & $<0.001$ \\
\hline
\end{tabular}

Data are mean \pm standard-deviation values.

$A \beta$ : $\beta$-amyloid, AD: Alzheimer's disease, CN: cognitively normal, iNPH: idiopathic normal-pressure hydrocephalus, p1: iNPH vs. AD, p2: iNPH vs. CN, p3: AD vs. CN, p-tau: phosphorylated tau, t-tau: total tau.

$\mathrm{AD}$ patients than in $\mathrm{CN}$ subjects $(p<0.032$; Fig. 1B), but it did not differ significantly between the iNPH and AD groups. Although the statistical significance was not high enough, $\mathrm{A} \beta 42$ and $\mathrm{A} \beta 40$ levels were numerically lower in iNPH patients than in $\mathrm{CN}$ subjects. The ratio of $\mathrm{A} \beta 42$ to $\mathrm{A} \beta 40$ levels was significantly lower in $\mathrm{AD}$ than in $\mathrm{iNPH}$ patients and $\mathrm{CN}$ subjects ( $p=0.001$ and $p=0.003$, respectively; Fig. $1 \mathrm{C}$ ), while it did not differ between the iNPH and CN groups.

We also assessed the CSF levels of t-tau and p-tau. We found that both t-tau and p-tau levels were significantly higher in $\mathrm{AD}$ than in iNPH and CN subjects ( $\mathrm{t}$-tau, $p<0.001$ and $p<$ 0.001 , respectively; $\mathrm{p}$-tau, $p<0.001$ and $p<0.001$, respectively; Fig. $1 \mathrm{D}$ and $\mathrm{E}$ ), while they did not differ between the iNPH and $\mathrm{CN}$ subjects. The CSF levels of $\mathrm{A} \beta 42$ and $\mathrm{A} \beta 40$ in each subject group as well as representative amyloid PET images of amyloid PET(+) AD and amyloid PET(-) iNPH patients are presented in Fig. 2.

\section{DISCUSSION}

In this study we found that the levels of both $A \beta 40$ and $A \beta 42$ in the CSF numerically decreased proportionately in amyloid PET(-) iNPH patients compared to CN subjects, although the statistical significance was not high enough. In contrast, amyloid $\mathrm{PET}(+) \mathrm{AD}$ patients showed a disproportionate de- crease in these levels. We also found that the A $\beta 42 / A \beta 40$ ratio can be used to successfully discriminate between iNPH and $\mathrm{AD}$. Finally, we have revealed differences in $\mathrm{t}$-tau and $\mathrm{p}$ tau levels between amyloid PET(+) AD patients and amyloid PET(-) iNPH patients. To our knowledge, this is the first study to investigate AD biomarkers in the CSF of amyloid PET(-) iNPH patients. We suggest that different mechanisms underlie the low $\mathrm{A} \beta 42$ observed in $\mathrm{iNPH}$ and AD patients.

There have been inconsistencies in the reported levels of $\mathrm{A} \beta 42$ in iNPH and AD patients, with some reports of overlapping levels ${ }^{7,20}$ but others of lower $\mathrm{A} \beta 42$ levels in $\mathrm{AD}$ than iNPH patients. ${ }^{5,8,10}$ These discrepancies might be due to the inclusion of different numbers of patients in the iNPH groups, possibly with coexisting AD and iNPH, since no amyloid PET analyses were performed in these previous studies. In our study we used amyloid PET to distinguish between amyloid PET(-) iNPH and amyloid PET(+) AD patients, and confirmed that the $A \beta 42$ levels are decreased in both patients, but more so in those with AD.

Our ELISA results demonstrated that the $A \beta 42 / A \beta 40$ ratio is significantly higher in iNPH than AD patients, with no differences found between the iNPH and $\mathrm{CN}$ subjects. In line with a previous study, ${ }^{8}$ these results suggest that $\mathrm{A} \beta 42$ and $A \beta 40$ levels are differentially decreased in $\mathrm{AD}$ and $\mathrm{iNPH}$ patients. The disproportionate decrease in $A \beta 42$ level has 

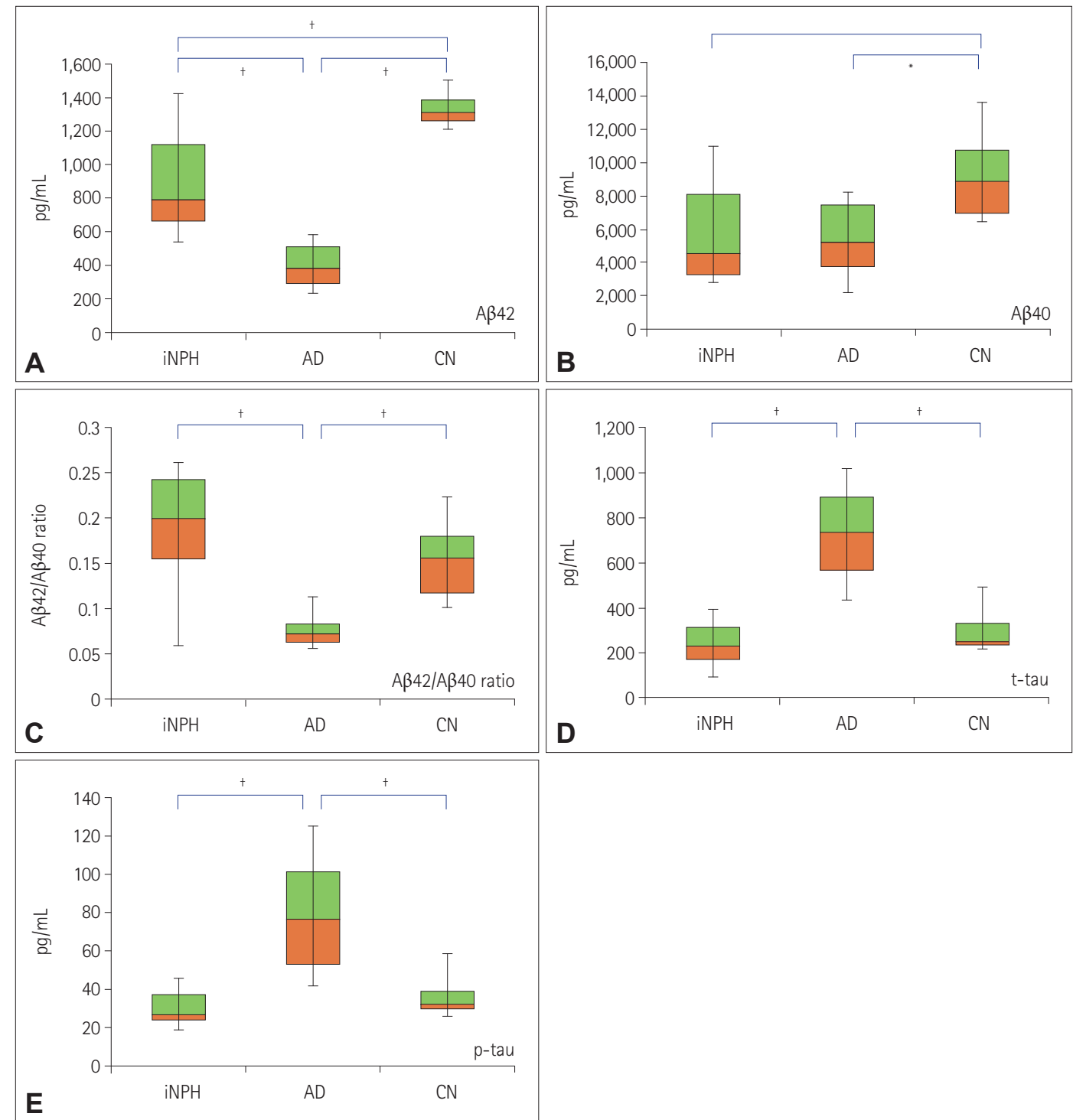

Fig. 1. $A D$ biomarker levels in the cerebrospinal fluid of $i N P H, A D$, and $C N$ subjects. Box plots of $A \beta 42(A), A \beta 40(B), A \beta 42 / A \beta 40$ ratio (C), t-tau (D), and $p$-tau (E). Each box plot shows the median, first and third quartiles, and range. ${ }^{*} p<0.05,{ }^{+} p<0.01$ (student $t$-test). A $\beta$ : $\beta$-amyloid, $A D$ : Alzheimer's disease, CN: cognitively normal, iNPH: idiopathic normal-pressure hydrocephalus, p-tau: phosphorylated tau, t-tau: total tau.

been shown to be a signature pattern in amyloid PET(+) AD patients, due to the higher tendency of $A \beta 42$ to be deposited in amyloid plaques. ${ }^{21}$ In contrast, the similarities in the $\mathrm{A} \beta 42 /$ $\mathrm{A} \beta 40$ ratio between the amyloid $\mathrm{PET}(-)$ iNPH and $\mathrm{CN}$ subjects suggest that there is no selective deposition of $A \beta 42$ in iNPH.

The distinct profile of CSF AD biomarkers in amyloid PET(-) iNPH patients in the present study differ from that of preclinical AD patients. Previous studies employing imaging and CSF AD biomarkers found that a decrease in $A \beta 42$ levels in amyloid PET(-) subjects was the earliest change before the amyloid deposition in the brain parenchyma of preclinical $\mathrm{AD}$ patients. ${ }^{22}$ Accordingly, the decreased $A \beta 42$ levels in iNPH have been attributed to an underlying early or preclinical $\mathrm{AD}$ pathology. However, a recent study investigating the pattern of CSF AD biomarkers in preclinical $\mathrm{AD}$ patients showed that the disproportionate decrease in $A \beta 42$ than $A \beta 40$ levels is still observed even in the early stages of preclinical $\mathrm{AD} .{ }^{23}$ Longitudinal observations of changes in the $A \beta 42 / A \beta 40$ ratio and the CSF t-tau and p-tau levels in amyloid PET(-) iNPH patients could elucidate the distinct CSF AD biomarker patterns during the initiation and progression of $\mathrm{AD}$ neuropathology in iNPH.

The mechanism underlying the low levels of $A \beta$ isoforms in iNPH has been highly controversial. Jeppsson et al. ${ }^{6}$ analyzed a panel of $A \beta$ isoforms $(A \beta 38, A \beta 40$, and $A \beta 42)$ and soluble APP (sAPP $\alpha$ and sAPP $\beta$ ) isoforms in iNPH and healthy individuals, and showed that all of these biomarkers were 


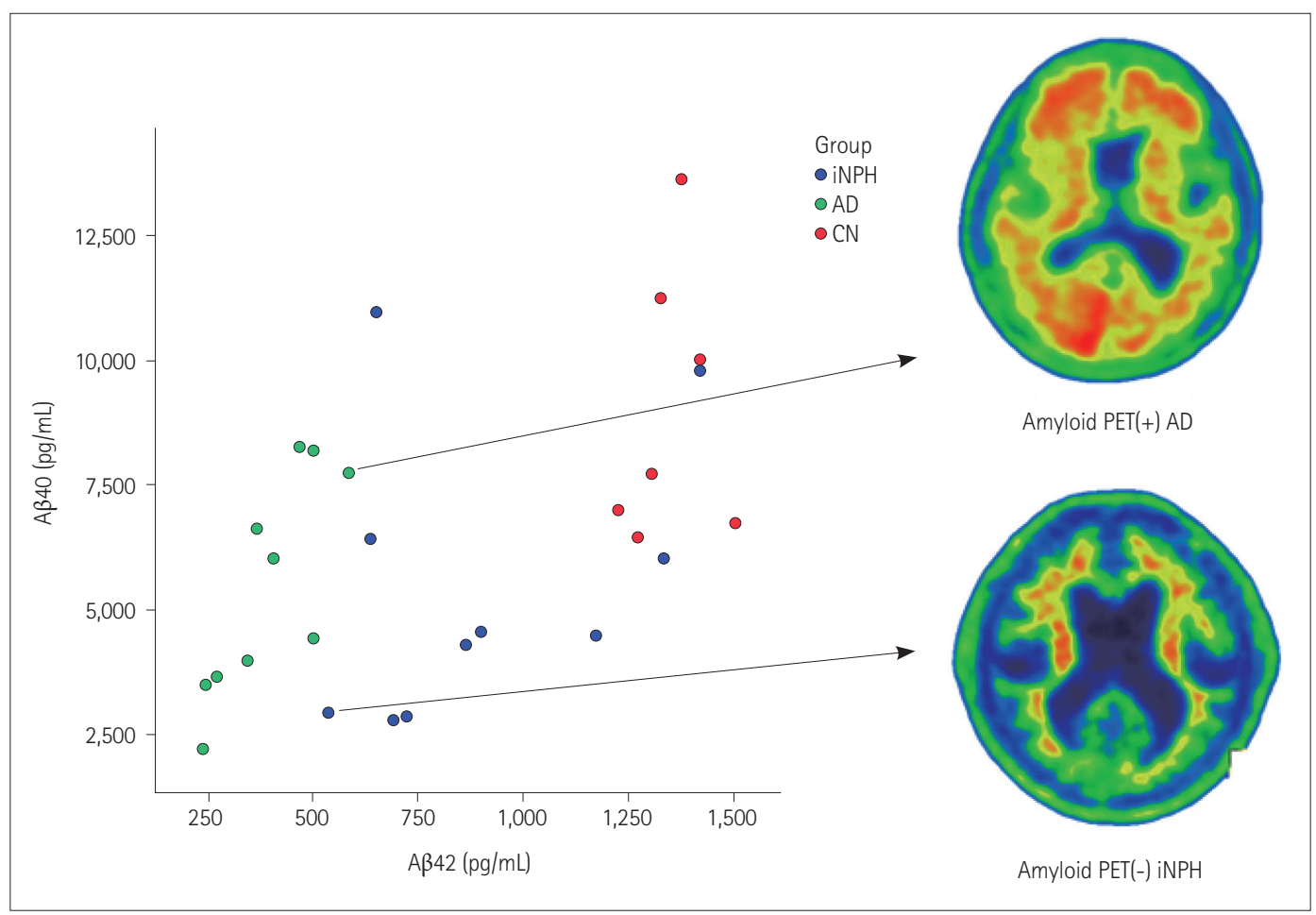

Fig. 2. Correlation between $A \beta 42$ and $A \beta 40$ levels in $i N P H, A D$, and $C N$ subjects. The scatter plot shows the correlation between $A \beta 42$ and $A \beta 40$ levels. Representative images from an amyloid PET(+) AD scan and an amyloid PET(-) iNPH scan are shown on the right. A $\beta$ : $\beta$-amyloid, AD: Alzheimer's disease, amyloid PET(+): amyloid positron-emission tomography-positive, amyloid PET(-): amyloid positron-emission tomography-negative, CN: cognitively normal, iNPH: idiopathic normal-pressure hydrocephalus.

reduced in iNPH presurgically but restored after shunt surgery. Those authors proposed that these findings could be explained by reduced periventricular amyloid metabolism and/or reduced clearance into the CSF. On the other hand, Graff-Radford suggested that glymphatic dysfunction could be causing the low CSF A $\beta$ isoforms. Indeed, there is a recent report of glymphatic dysfunction in iNPH patients, which was detected by using glymphatic MRI after injection with an intrathecal contrast agent. ${ }^{9,24}$ Meanwhile, transient glymphatic dysfunction caused by sleep deprivation has been reported to increase CSF A $\beta$ levels; however, little is known about the chronic glymphatic dysfunction in iNPH patients. ${ }^{25,26} \mathrm{~A}$ longitudinal serial evaluation of glymphatic function in association with CSF AD biomarkers is therefore required to elucidate how it affects the levels of these biomarkers.

A meta-analysis found that tau levels were lower in NPH than in $\mathrm{AD},{ }^{10}$ which is consistent with our results. Tau levels in our iNPH patients were significantly lower than those in the $\mathrm{AD}$ patients and numerically lower than those in $\mathrm{CN}$ subjects. Previous studies have found that the level of tau increases with age. ${ }^{27,28}$ In our study, the patients with NPH were older than the $\mathrm{AD}$ and $\mathrm{CN}$ subjects, but lower levels of tau suggest that the pathophysiology differs between $\mathrm{AD}$ and NPH. Our study supports that reduced clearance from extracellular fluid and decreased brain metabolism of the peri- ventricular zone in iNPH may contribute to this phenomenon of reduced CSF t-tau and p-tau levels in iNPH. ${ }^{9}$

The present study was subject to some limitations. Firstly, the sample was relatively small. Secondly, iNPH and AD patients were prospectively recruited from a single hospital, while the $\mathrm{CN}$ subjects were recruited from another hospital, which could have caused selection bias. Furthermore, the CN subjects were enrolled from the community, which meant their brain MRI and amyloid PET data were not available. However, we could measure consistent values for the CSF AD biomarkers, indicating the absence of $\mathrm{AD}$ pathology in these subjects. Finally, our $\mathrm{AD}$ patients had early-onset $\mathrm{AD}$ and the pathomechanism of amyloidopathy of early-onset AD differs from that of late-onset AD. Further studies with larger samples and a longitudinal design are therefore required.

\section{Conflicts of Interest}

The authors have no potential conflicts of interest to disclose.

\section{Acknowledgements}

This research was supported by a grant of the Korea Health Technology R\&D Project through the Korea Health Industry Development Institute (KHIDI), funded by the Ministry of Health \& Welfare, Republic of Korea (grant number: HI18C0479) to S.Y.M; by a grant of the National Research Foundation of Korea (NRF) funded by the Ministry of Science, ICT (NRF2016R1C1B2010206) to J.C. 


\section{REFERENCES}

1. Adams RD, Fisher CM, Hakim S, Ojemann RG, Sweet WH. Symptomatic occult hydrocephalus with "normal" cerebrospinal-fluid pressure. A treatable syndrome. N Engl J Med 1965;273:117-126.

2. Leinonen V, Koivisto AM, Savolainen S, Rummukainen J, Tamminen JN, Tillgren T, et al. Amyloid and tau proteins in cortical brain biopsy and Alzheimer's disease. Ann Neurol 2010;68:446-453.

3. Golomb J, Wisoff J, Miller DC, Boksay I, Kluger A, Weiner H, et al. Alzheimer's disease comorbidity in normal pressure hydrocephalus: prevalence and shunt response. J Neurol Neurosurg Psychiatry 2000;68: 778-781.

4. Blennow K, Hampel H. CSF markers for incipient Alzheimer's disease. Lancet Neurol 2003;2:605-613.

5. Lim TS, Choi JY, Park SA, Youn YC, Lee HY, Kim BG, et al. Evaluation of coexistence of Alzheimer's disease in idiopathic normal pressure hydrocephalus using ELISA analyses for CSF biomarkers. BMC Neurol 2014;14:66.

6. Jeppsson A, Zetterberg H, Blennow K, Wikkelsø C. Idiopathic normal-pressure hydrocephalus: pathophysiology and diagnosis by CSF biomarkers. Neurology 2013;80:1385-1392.

7. Kapaki EN, Paraskevas GP, Tzerakis NG, Sfagos C, Seretis A, Kararizou E, et al. Cerebrospinal fluid tau, phospho-tau ${ }_{181}$ and $\beta$-amyloid ${ }_{1-42}$ in idiopathic normal pressure hydrocephalus: a discrimination from Alzheimer's disease. Eur J Neurol 2007;14:168-173.

8. Jingami N, Asada-Utsugi M, Uemura K, Noto R, Takahashi M, Ozaki A, et al. Idiopathic normal pressure hydrocephalus has a different cerebrospinal fluid biomarker profile from Alzheimer's disease. $J$ Alzheimers Dis 2015;45:109-115.

9. Graff-Radford NR. Alzheimer CSF biomarkers may be misleading in normal-pressure hydrocephalus. Neurology 2014;83:1573-1575.

10. Chen Z, Liu C, Zhang J, Relkin N, Xing Y, Li Y. Cerebrospinal fluid $\mathrm{A} \beta 42, \mathrm{t}$-tau, and $\mathrm{p}$-tau levels in the differential diagnosis of idiopathic normal-pressure hydrocephalus: a systematic review and meta-analysis. Fluids Barriers CNS 2017;14:13.

11. Relkin N, Marmarou A, Klinge P, Bergsneider M, Black PM. Diagnosing idiopathic normal-pressure hydrocephalus. Neurosurgery 2005;57: S4-16.

12. Larsson A, Wikkelsö C, Bilting M, Stephensen H. Clinical parameters in 74 consecutive patients shunt operated for normal pressure hydrocephalus. Acta Neurol Scand 1991;84:475-482.

13. Krauss JK, Regel JP, Vach W, Jüngling FD, Droste DW, Wakhloo AK. Flow void of cerebrospinal fluid in idiopathic normal pressure hydrocephalus of the elderly: can it predict outcome after shunting? Neurosurgery 1997;40:67-73.

14. McKhann G, Drachman D, Folstein M, Katzman R, Price D, Stadlan EM. Clinical diagnosis of Alzheimer's disease: report of the NINCDSADRDA Work Group under the auspices of Department of Health and Human Services Task Force on Alzheimer's Disease. Neurology 1984; 34:939-944.

15. Moon SY, Na DL, Seo SW, Lee JY, Ku BD, Kim SY, et al. Impact of white matter changes on activities of daily living in mild to moderate dementia. Eur Neurol 2011;65:223-230.

16. Ahn HJ, Chin J, Park A, Lee BH, Suh MK, Seo SW, et al. Seoul Neuropsychological Screening Battery-dementia version (SNSB-D): a useful tool for assessing and monitoring cognitive impairments in dementia patients. J Korean Med Sci 2010;25:1071-1076.

17. Sabri O, Seibyl J, Rowe C, Barthel H. Beta-amyloid imaging with florbetaben. Clin Transl Imaging 2015;3:13-26.

18. Tzourio-Mazoyer N, Landeau B, Papathanassiou D, Crivello F, Etard $\mathrm{O}$, Delcroix N, et al. Automated anatomical labeling of activations in SPM using a macroscopic anatomical parcellation of the MNI MRI single-subject brain. Neuroimage 2002;15:273-289.

19. Choi WH, Um YH, Jung WS, Kim SH. Automated quantification of amyloid positron emission tomography: a comparison of PMOD and MIMneuro. Ann Nucl Med 2016;30:682-689.

20. Santangelo R, Cecchetti G, Bernasconi MP, Cardamone R, Barbieri A, Pinto P, et al. Cerebrospinal fluid amyloid- $\beta 42$, total tau and phosphorylated tau are low in patients with normal pressure hydrocephalus: analogies and differences with Alzheimer's disease. J Alzheimers Dis 2017;60:183-200.

21. Lewczuk P, Matzen A, Blennow K, Parnetti L, Molinuevo JL, Eusebi P, et al. Cerebrospinal fluid $A \beta 42 / 40$ corresponds better than $A \beta 42$ to amyloid PET in Alzheimer's disease. J Alzheimers Dis 2017;55:813822.

22. Palmqvist S, Mattsson N, Hansson O; Alzheimer's Disease Neuroimaging Initiative. Cerebrospinal fluid analysis detects cerebral amyloid- $\beta$ accumulation earlier than positron emission tomography. Brain 2016; 139:1226-1236.

23. Toledo JB, Bjerke M, Da X, Landau SM, Foster NL, Jagust W, et al. Nonlinear association between cerebrospinal fluid and florbetapir F-18 $\beta$-amyloid measures across the spectrum of Alzheimer disease. JAMA Neurol 2015;72:571-581.

24. Sutphen CL, Jasielec MS, Shah AR, Macy EM, Xiong C, Vlassenko AG, et al. Longitudinal cerebrospinal fluid biomarker changes in preclinical Alzheimer disease during middle age. JAMA Neurol 2015;72: 1029-1042.

25. Xie L, Kang H, Xu Q, Chen MJ, Liao Y, Thiyagarajan M, et al. Sleep drives metabolite clearance from the adult brain. Science 2013;342: 373-377.

26. Ju YS, Ooms SJ, Sutphen C, Macauley SL, Zangrilli MA, Jerome G, et al. Slow wave sleep disruption increases cerebrospinal fluid amyloid- $\beta$ levels. Brain 2017;140:2104-2111.

27. Paternicò D, Galluzzi S, Drago V, Bocchio-Chiavetto L, Zanardini R, Pedrini L, et al. Cerebrospinal fluid markers for Alzheimer's disease in a cognitively healthy cohort of young and old adults. Alzheimers Dement 2012;8:520-527.

28. Sjögren M, Vanderstichele H, Agren H, Zachrisson O, Edsbagge M, Wikkelsø C, et al. Tau and $A \beta 42$ in cerebrospinal fluid from healthy adults 21-93 years of age: establishment of reference values. Clin Chem 2001;47:1776-1781. 